\title{
Wound fluids affect miR-21, miR-155 and miR-221 expression in breast cancer cell lines, and this effect is partially abrogated by intraoperative radiation therapy treatment
}

\author{
KAROLINA ZALESKA ${ }^{1}$, ANNA PRZYBYŁA ${ }^{2}$, KATARZYNA KULCENTY ${ }^{1,3}$, MATEUSZ WICHTOWSKI $^{4}$, \\ ANDRZEJ MACKIEWICZ ${ }^{2,5}$, WIKTORIA SUCHORSKA ${ }^{1,3}$ and DAWID MURAWA ${ }^{4,6}$ \\ ${ }^{1}$ Radiobiology Laboratory, Greater Poland Cancer Centre, 61-866 Poznań; \\ ${ }^{2}$ Department of Cancer Immunology, Chair of Medical Biotechnology; ${ }^{3}$ Department of Electoradiology, \\ Poznań University of Medical Sciences, 61-701 Poznań; ${ }^{4}$ First Clinic of Surgical Oncology and General Surgery; \\ ${ }^{5}$ Department of Cancer Diagnostics and Immunology, Greater Poland Cancer Centre, 61-866 Poznań; \\ ${ }^{6}$ Research and Development Centre, Regional Specialist Hospital in Wrocław, 51-124 Wrocław, Poland
}

Received February 20, 2017; Accepted July 13, 2017

DOI: $10.3892 / \mathrm{ol} .2017 .6718$

\begin{abstract}
Breast cancer is the most common malignant disease occurring in women. Conservative breast cancer surgery followed by radiation therapy is currently the standard treatment for this type of cancer. The majority of metastases occur within the scar, which initiated a series of studies. As a result, clinical trials aimed to assess whether localized radiotherapy, as intraoperative radiotherapy (IORT), may more effective in inhibiting the formation of local recurrence compared with the standard postoperative whole breast radiotherapy. The present study determined the role of postoperative wound fluids (WFs) from patients diagnosed with breast cancer subsequent to breast conserving surgery or breast conserving surgery followed by IORT on the expression of three microRNAs (miRNAs), consisting of miR-21, miR-155 and miR-221, in distinct breast cancer cell lines that represent the general subtypes of breast cancer. It was determined that the miRNAs responsible for breast cancer progression, induction of tumorigenesis and enrichment of the cancer stem cell phenotype, which is responsible for resistance to tumor therapy, were highly upregulated in the human epidermal growth factor receptor 2-positive breast cancer SK-BR-3 cell line following stimulation with WFs. It is worth emphasizing, that those changes were more significant in WFs collected from patients after surgery alone. The BT-549 cell line showed altered expression only of miR-155
\end{abstract}

Correspondence to: Mrs. Karolina Zaleska, Radiobiology Laboratory, Greater Poland Cancer Centre, Garbary 15 Street, 61-866 Poznań, Poland

E-mail: zaleska.kar@gmail.com

Key words: breast cancer, intraoperative radiotherapy, surgical wound fluids, microRNA following incubation with WFs. Notably, this change was not associated with IORT. Additionally, it was indicated that both WFs and RT-WF strongly downregulated the expression of miR-21, miR-155 and miR-221 in basal/epithelial and luminal subtypes of breast cancer. It was concluded that the present study contributes to an increased understanding of the role of surgical WFs and IORT treatment in the regulation of miRNA expression. This may enable the development of the current knowledge of breast cancer biology subsequent to IORT treatment and substantially to improve the therapy in the future.

\section{Introduction}

Breast cancer is one of the most frequent malignances and the second most common cause of cancer-associated mortality in women around the world. The morbidity rate of breast cancer has gradually increased in recent decades (1). Treatment includes surgical intervention, radiotherapy (RT), systemic treatment with cytotoxic chemotherapy, hormone therapy, biological therapy, or a combination of all these therapies (2). Subsequent to breast-conserving surgery, the tumor bed represents the region with the highest probability of recurrence $(\leq 90 \%)(3,4)$. It was determined that the most effective strategy is intraoperative RT (IORT) $(5,6)$. During IORT, a $10-$ Gy electron-boost is delivered within breast-conserving treatment $(\mathrm{BCT})$, which results in notably low local recurrence rates (7). It is known that post-operative wound fluids (WFs) collected from breast cancer patients are able to stimulate in vitro growth of breast cancer cells and are potent activators of the signal transducer and activator of transcription factors, due to enriched composition of cytokines and growth factors related to wound healing. WFs have an important role in breast cancer cell proliferation, migration and survival $(8,9)$. Based on these findings and having the knowledge that IORT contributes to low recurrence rates (5-7), attempts were made to determine whether the post-surgery wound fluid after intraoperative irradiation, may modify the wound microenvironment, making it less favorable for cancer cell growth and 
invasion (8). IORT is the key factor that affects the wound healing microenvironment, which affects cancer cell biology by decreasing the growth and invasion potential. A study by Belletti et al (8) demonstrated that WFs from patients treated with targeted IORT significantly modify the proteomic expression profile of molecules associated with tumor growth and motility. Additionally, the effect of surgical wounds after intraoperative radiotherapy on the cancer stem cell (CSC) phenotype was previously investigated in a panel of human breast cancer cell lines (10). It was found that WF and WF after IORT treatment affects the putative stem cell phenotype in breast cancer cell lines. Additionally, this stimulatory effect was decreased in WF after IORT treatment.

In breast cancer, a number of microRNAs (miRNAs) have been identified as tumor suppressors or oncogenes and have been characterized as critical regulators of tumor initiation, metastasis and chemoresistance (11). miRNAs have also emerged as critical regulators of drug resistance that act by modulating the epithelial-to-mesenchymal transition (EMT) and cancer-associated immune responses (12). miRNAs are molecules that contribute to modulating signaling pathways subsequent to radiation exposure and have emerged as a potential therapeutic target or biomarker in the radiation response of cancer (13). However, there is insufficient data on the effect of IORT and the wound-healing process on miRNA regulation in the breast cancer cells present in that microenvironment.

Due to evidence that post-surgical WFs after IORT can modify the proteomic expression profile, and thus the wound microenvironment, the aim of the present study was to determine whether post-surgery WFs had an effect on the miRNA expression level in breast cancer cells, and if those changes are associated with intraoperative radiation therapy.

\section{Materials and methods}

Surgical WF collection. Postoperative WFs were collected from breast cancer patients that underwent surgical treatment in Greater Poland Cancer Centre in Poznan, Poland. Wound fluids were collected between November 2013 and January 2015. Following resection of the tumor (wide local excision), one group of patients underwent IORT up to a dose of 10 Gy per tumor bed (Boost) (RT-WF group); the second group of patients did not receive IORT (WF group). The clinical characteristics of each group are presented in Table I. The follow-up examination was scheduled 7 days after surgery at the Greater Poland Cancer Centre in Poznan, Poland. The patients underwent the ultrasonography and were assessed for the presence of fluid in the tumor bed. WFs were collected by means of percutaneous aspiration. Fluids were centrifuged for $25 \mathrm{~min}$ at $300 \times \mathrm{g}$ at $4^{\circ} \mathrm{C}$, sterile filtered and stored at $-80^{\circ} \mathrm{C}$. The study was approved by the Bioethics Committee of Poznań University of Medical Sciences (Poznań, Poland). Informed consent was obtained from all patients.

Cell culture. Experiments were performed on four breast cancer cell lines, consisting of MDA-MB-468 [Er/PgR-; human epidermal growth factor receptor 2 (HER2)/Neu-], BT-549 (Er/PgR-; HER2/Neu-), MCF-7 (Er/PgR+; HER2/Neu-) and
SK-BR-3 (Er/PgR-; HER2/Neu+). MDA-MB-468 and BT-549 are defined as triple-negative breast cancer (TNBC). They were chosen due to their distinct molecular profile $(14,15)$. All cell lines were obtained from the American Type Culture Collection (Manassas, VA, USA) and maintained according to the supplier's instructions. Cells were grown at $37^{\circ} \mathrm{C}$ in $5 \%$ $\mathrm{CO}_{2}$. Cells were seeded on 6-cm Petri dishes and incubated with standard medium overnight $24 \mathrm{~h}$ prior to experiments. Subsequently, culture medium was changed to fresh medium containing $10 \%$ RT-WF or $10 \%$ WF. The cells were incubated in standard culture conditions $\left(37^{\circ} \mathrm{C}\right.$ in an atmosphere containing $5 \% \mathrm{CO}_{2}$ ) for 4 days. Control cells (untreated cells cultured in the same conditions) were harvested in standard medium under the same conditions.

RNA isolation. Total RNA from 20 samples for each cell line (10 samples per group; RT-WF or WF) was extracted with TRIzol reagent (Sigma-Aldrich; Merck KGaA, Darmstadt, Germany), according to the manufacturer's instructions. Cells were lysed using TRIzol reagent. For the miRNA normalization process, $3.5 \mu \mathrm{l}$ of miRNeasy Serum/Plasma Spike-In Control working solution $\left(1.6 \times 10^{8}\right.$ copies $/ \mu 1$; Qiagen, Hilden, Germany) was added to each sample. The RNA eluted in diethylpyrocarbonate-treated $\mathrm{H}_{2} \mathrm{O}$ was stored at $-80^{\circ} \mathrm{C}$ until further analyses.

Reverse transcription $(R T)$. The $\mathrm{RT}$ reactions were performed using the Taq-Man Reverse Transcription kit and miRNA-specific stem-loop primers: Human (hsa-) mir-21-5p (assay ID, 000397), hsa-mir-155-5p (assay ID, 002623), hsa-mir-221 (assay ID, 000524), cel-miR-39 (reference gene; assay ID, 000200; Applied Biosystems; Thermo Fisher Scientific, Inc., Waltham, MA, USA). Each reaction consisted of $10 \mathrm{ng}$ miRNA, 10X RT buffer, $20 \mathrm{U} / \mu 1$ RNase inhibitor, 5X TaqMan miRNA RT primer, $100 \mathrm{mmol} / \mathrm{l} \mathrm{dNTP}$ and $50 \mathrm{U} / \mu 1$ MultiScribe Reverse Transcriptase. The RT reaction was performed using a Veriti ${ }^{\circledR}$ 96-Well Fast Thermal Cycler (Applied Biosystems; Thermo Fisher Scientific, Inc.) at $16^{\circ} \mathrm{C}$ for $30 \mathrm{~min}, 42^{\circ} \mathrm{C}$ for $30 \mathrm{~min}$ and $85^{\circ} \mathrm{C}$ for $5 \mathrm{~min}$.

Quantitative polymerase chain reaction ( $q P C R)$. qPCR analysis of hsa-miR-21, hsa-miR-155, hsa-miR-221 and miRNeasy Serum/Plasma Spike-In Control $\left(1.6 \times 10^{8}\right.$ copies $\left./ \mu 1\right)$ as a reference, was performed using a miR-specific TaqMan MicroRNA Assay kit (Applied Biosystems; Thermo Fisher Scientific, Inc.). Analyzed miRNA primers and reference miRNA primers used were as aforementioned for reverse transcription (miRNA-specific stem-loop primers; Applied Biosystems; Thermo Fisher Scientific, Inc.). The amplification protocol was as follows: $95^{\circ} \mathrm{C}$ for $10 \mathrm{~min}$, followed by 50 cycles of $95^{\circ} \mathrm{C}$ for $15 \mathrm{sec}, 60^{\circ} \mathrm{C}$ for $1 \mathrm{~min}$, and $4^{\circ} \mathrm{C}$ for $30 \mathrm{sec}$. The qPCR reactions were performed in triplicate according to the manufacturer's instructions using LightCycler480 (Roche Diagnostics, Basel, Switzerland). The fold-differences in miRNA expression between the samples were calculated using the comparative $\mathrm{Cq}\left(2^{-\Delta \Delta \mathrm{Cq}}\right)$ method (16).

Statistical analysis. Quantitative differential expression of miRNAs between patients was calculated as $2^{-\Delta \Delta C q}$. For the statistical analysis the GraphPad Prism program (version 6; 
Table I. Histopatological classification of patients.

\begin{tabular}{lcr}
\hline & \multicolumn{2}{c}{ Treatment group, $\%$} \\
\cline { 2 - 3 } Clinicopathological features & RT-WF & WF \\
\hline Total, n & 22.0 & 20.0 \\
Age at diagnosis, years \pm SD & $60.1 \pm 9.6$ & $55.0 \pm 10.2$ \\
Histological type & & \\
Ductal & 59.1 & 65.0 \\
Lobular & 22.7 & 35.0 \\
Other & 18.2 & 0.0 \\
ER status & & \\
Positive & 100.0 & 95.0 \\
Negative & 0.0 & 5.0 \\
HER2 status & & \\
Positive & 0.0 & 10.0 \\
Negative & 100.0 & 90.0 \\
Molecular classification & & \\
Triple negative & 0.0 & 5.0 \\
Non-triple negative & 100.0 & 95.0 \\
Histological grade & & 25.0 \\
G1 & 40.9 & \\
G2 & 31.8 & \\
G3 & 27.3 & \\
\hline
\end{tabular}

SD, standard deviation; WF, post-operative wound fluid; RT-WF, post-operative and post-intraoperative radiotherapy wound fluid; ER, estrogen receptor; HER2, human epidermal growth factor receptor 2.

GraphPad Software, Inc., La Jolla, CA, USA) was used. Data were examined using one-way analysis of variance with Tukey's post hoc test. $\mathrm{P}<0.05$ was considered to indicate a statistically significant difference.

\section{Results}

Evaluation of miRNA expression in breast cancer cell lines. The expression level of three different miRNAs, consisting of miR-21, miR-155 and miR-221, was assessed by RT-qPCR analysis in cell lines from 4 subtypes of breast cancer, consisting of the basal/mesenchymal BT-549, basal/epithelial MDA-MB-468, HER2-overexpressed SB-BR-3 and luminal MCF7 cell line (Fig. 1). The expression profiles of miR-21 and miR-221 were similar in all analyzed cell lines. BT-549, MDA-MB-468 and MCF7 cells showed similar expression levels of miR-21 and miR-221. Similar patterns were also observed in SK-BR-3 cell line; however, the expression of those miRNAs was much lower. miR-155 represented the lowest expression profile among all analyzed cell lines. The expression of all analyzed miRNAs was lowest in the SK-BR3 cell line.

Expression of miR-21, miR-155 and miR-221 is affected by WFs in different subtypes of breast cancer cells. The present study confirmed that all the miRNAs were differentially expressed in breast cancer cells (Fig. 1). To establish the effect of WFs on the expression of the 3 analyzed miRNAs, cells were incubated with $10 \%$ of WF or RT-WF and harvested after 4 days. The levels of miRNA expression were evaluated using the TaqMan MicroRNA Assay.

miR-21 expression. Breast cancer cells from 4 subtypes of breast cancer demonstrated an altered expression level of miR-21 after 4-day stimulation with $10 \%$ WF or RT-WF, and the results varied markedly between each cell line (Fig. 2). The basal/mesenchymal BT-549 cell line did not exhibit any changes in the expression of miR-21 following incubation with WF or RT-WF (WF, 1.04 \pm 0.26 ; RT-WF, 1.04 \pm 0.24 ). Notably, the basal/epithelial MDA-MB-468 and luminal MCF7 cell lines exhibited a similar expression profile of miR-21. Following incubation with WF and RT-WF, the expression of analyzed miRNA was significantly decreased $(\mathrm{P}<0.0001)$. No changes were observed between the WF and RT-WF groups in the MDA-MB-468 cell line (WF, 0.12 \pm 0.05 ; RT-WF, $0.11 \pm 0.03$ ). By contrast, the RT-WF group exhibited slightly increased expression of miR-21 compared with the WF group in the MCF7 cell line; however, those results were not statistically significant (WF, 0.15 \pm 0.04 ; RT-WF, 0.21 \pm 0.07 ). In contrast to the aforementioned cell line, the HER-positive SK-BR-3 cell line reacted differently to WF and RT-WF. Both WFs significantly increased $\mathrm{miR}-21$ expression (WF, $\mathrm{P}=0.005$; RT-WF, $\mathrm{P}=0.02)$; however, the stimulation with WF was markedly higher (WF, 4.47 \pm 0.9 ; RT-WF, 3.86 \pm 0.4 ).

miR-155 expression. As shown in Fig. 1, miR-155 exhibits the lowest expression in breast cell lines in comparison with the two other analyzed miRNAs. In the BT-549 cell line, expression of miR-155 was significantly decreased $(\mathrm{P}=0.0003)$ after incubation with WF and RT-WF in comparison to the control. However, differences between cells exposed to WF or RT-WF were not observed (WF, 0.43 \pm 0.05 ; RT-WF, $0.38 \pm 0.08$; Fig. 3). Similar results were also identified for the MDA-MB-468 cell line $(\mathrm{P}<0.0001)$. In contrast to the BT-549 cell line, in MDA-MB-468 cells a slight increase in miR-155 expression was observed following incubation with RT-WF (compared with WF); however, this was not statistically significant (WF, 0.22 \pm 0.1 ; RT-WF, $0.24 \pm 0.15$ ). By contrast, the HER2-overexpressing SK-BR-3 breast cancer cell line exhibits the most pronounced differences in miR-155 expression. WF causes the highest upregulation of miR-155 (11.84 \pm 5.2 ; $\mathrm{P}=0.012$ ) in comparison to the control and RT-WF groups. Increased expression of miR-155 was also observed following exposure to RT-WF, but at a significantly lower level (3.67 \pm 1.3 ; $\mathrm{P}=0.024)$. Additionally, the MCF7 cell line exhibited significantly decreased miR-155 expression $(\mathrm{P}<0.0001)$ and this decrease was more noticeable in the WF group compared with the RT-WF group $(\mathrm{P}=0.012$; WF, $0.05 \pm 0.01$; RT-WF, $0.09 \pm 0.03)$.

miR-221 expression. miR-221 expression shows a similar expression profile to miR-21 expression subsequent to $\mathrm{WF}$ and RT-WF supplementation of culture medium (Fig. 4). In the BT-549 cell line, no changes in miRNA expression were observed subsequent to WF or RT-WF stimulation (WF, $1.02 \pm 0.27$; RT-WF, $1.09 \pm 0.3$ ). Incubation of MDA-MB-468 


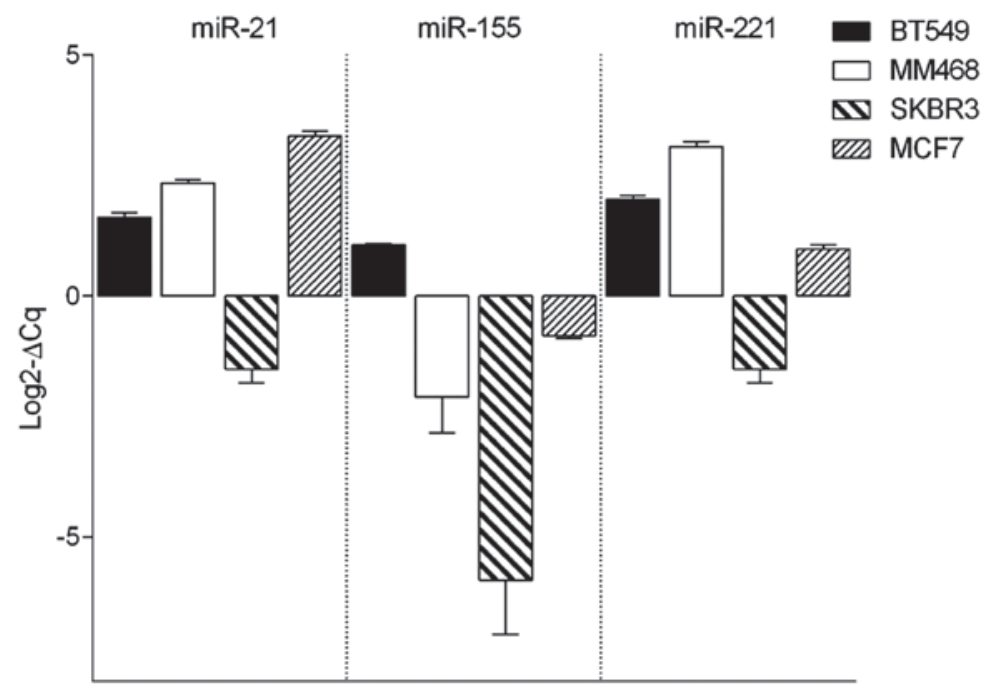

Figure 1. The evaluation of microRNA expression in different breast cancer cell lines. The expression of miR-21, miR-155 and miR-221 was evaluated in four breast cancer cell lines corresponding to four histopathological and molecular profiles. While miR-21 and miR-221 show high expression in three of the four analyzed cell lines, with the exception of the human epidermal growth factor receptor 2-positive SK-BR-3 cell line, miR-155 expression was extremely low. Graphs represent mean of $\log 2-\Delta \mathrm{Cq}$ of three experimental replicates.
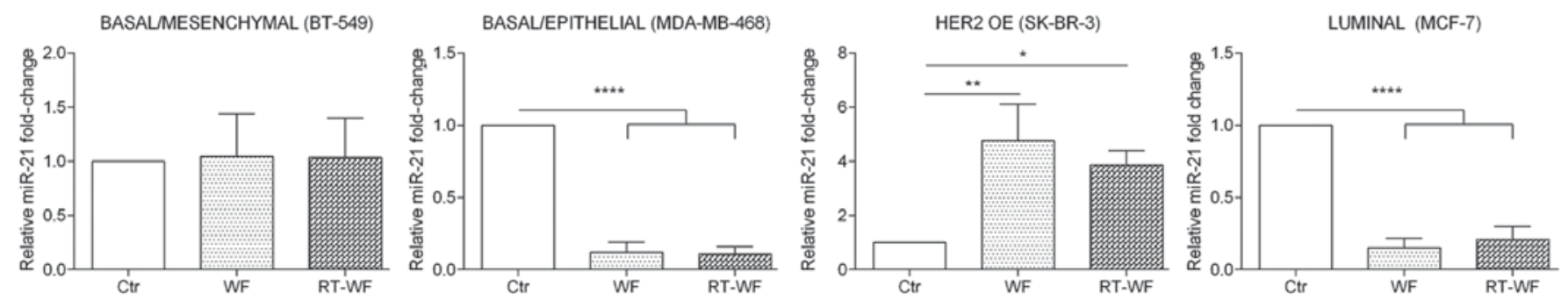

Figure 2. Expression of miR-21 is affected by wound fluids in cells from the basal/epithelial, HER2 OE and luminal subtypes of breast cancer. Expression of miR-21 did not change in the BT-549 cell line, and was decreased in the MDA-MB-468 and MCF7 cell lines following incubation with WF and RT-WF. In the SK-BR-3 cell line, miR-21 expression was increased after WF and RT-WF stimulation. Graphs show the relative mRNA fold change normalized to untreated cell lines. All samples were assessed in triplicate and analyzed using one-way analysis of variance with Tukey's post-hoc test. P $<0.05$ was considered to indicate a statistically significant difference. HER2 OE, human epidermal growth factor receptor 2 overexpression; WF, post-operative wound fluid; RT-WF, post-operative and post-intraoperative radiotherapy wound fluid. ${ }^{*} \mathrm{P}<0.05 ;{ }^{* *} \mathrm{P}<0.01 ;{ }^{* * *} \mathrm{P}<0.001$.
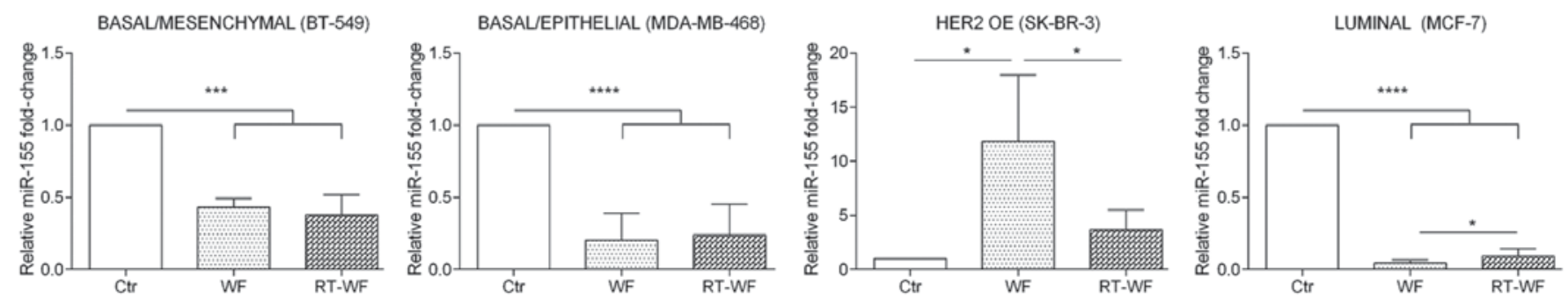

Figure 3. Expression of miR-155 is highly affected by wound fluids in all subtypes of analyzed breast cancer cells. Expression of miR-155 was decreased significantly in the BT-549, MDA-MB-468 and MCF7 cell lines. In the MCF7 cell line, an increase in miR-155 expression following RT-WF stimulation was also identified compared with WF stimulation. By contrast, the miR-155 expression level was increased in the SK-BR-3 cell line stimulated with WF. Only low expression stimulation was observed in the RT-WF group, but it was not statistically significant compared with the control. Graphs show the relative mRNA fold change normalized to untreated cell lines. All the samples were assessed in triplicate and analyzed using one-way analysis of variance with Tukey's post-hoc test. $\mathrm{P}<0.05$ was considered to indicate a statistically significant difference. HER2 OE, human epidermal growth factor receptor 2 overexpression; WF, post-operative wound fluid; RT-WF, post-operative and post-intraoperative radiotherapy wound fluid. ${ }^{*} \mathrm{P}<0.05 ;{ }^{* *} \mathrm{P}<0.01 ;{ }^{* * *} \mathrm{P}<0.001$.

and MCF7 cells with WF and RT-WF resulted in a prominent decrease in miR-221 expression $(\mathrm{P}<0.0001)$. For MDA-MB-468 cells, no statistical changes were observed between the WF and RT-WF groups (WF, 0.11 \pm 0.06 ; RT-WF, $0.07 \pm 0.03)$; however, non-significant decreased expression of miR-221 in the RT-WF group was observed. In contrast to other cell lines, in SK-BR-3 cells the miR-221 expression was increased following incubation with both WFs; however, statistical significance was observed only in WF-treated cells $(\mathrm{P}=0.0015$; WF, 4.76 \pm 0.9 ; RT-WF, 3.42 \pm 0.7$)$. 

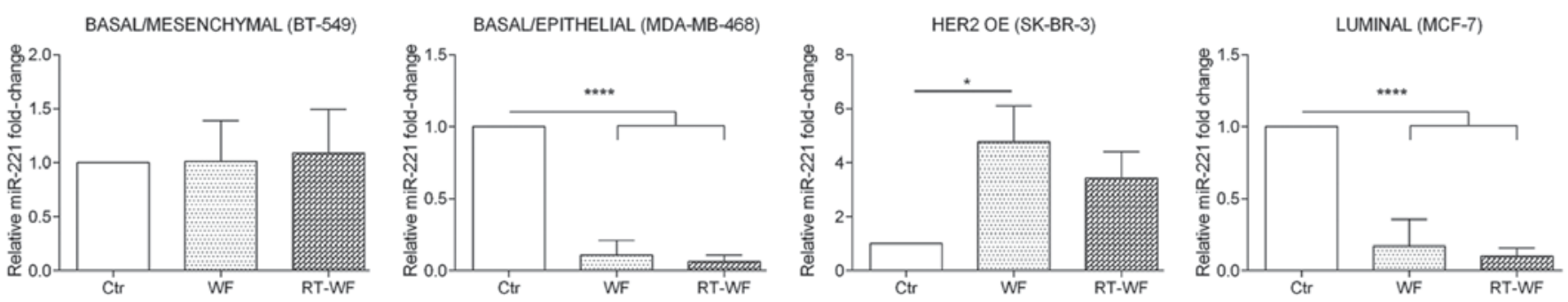

Figure 4. Expression of miR-221 is affected by wound fluids in basal/epithelial, HER2 OE and luminal subtype of breast cancer cells. The expression of miR-221 was not changed in the BT-549 cell line. The miR-221 expression level was decreased in MDA-MB-468 and MCF7 cells after incubation with WF and RT-WF. miR-221 expression was also increased in the SK-BR-3 cell line stimulated with WF. Only low expression stimulation was seen in RT-WF group, but it was not statistically significant compared with the control cells. Graphs show the relative mRNA fold change normalized to untreated cell lines. All the samples were assessed in triplicate and analyzed using one-way analysis of variance with Tukey's post-hoc test. $\mathrm{P}<0.05$ was considered to indicate a statistically significant difference. HER2 OE, human epidermal growth factor receptor 2 overexpression; WF, post-operative wound fluid; RT-WF, post-operative and post-intraoperative radiotherapy wound fluid; $\mathrm{Ctr}$, control. ${ }^{*} \mathrm{P}<0.05 ;{ }^{* *} \mathrm{P}<0.01 ;{ }^{* * *} \mathrm{P}<0.001$.

\section{Discussion}

Surgery itself can activate numerous inflammatory responses that are known to modify the growth kinetics of breast cancer micro-metastasis, suggesting that it can be also a factor in local recurrence or metastasis development (17-19).

It was shown that not only surgery, but also the post-surgery WF drainage collected from breast cancer patients can have a prominent role in breast cancer cell proliferation, motility and survival $(8,20,21)$. Thus, it is possible that modification of the local microenvironment caused by surgery may alter the growth kinetics of cancer cells, supporting the 'seed and soil' hypothesis first proposed by Sir Stephen Paget to explain the pathogenesis of cancer metastasis $(22,23)$. Additionally, it has been proposed that radiotherapy may affect not only breast cancer cell survival, but may also change the cell phenotype, physical interactions, signaling between cells and thus the entire tumor microenvironment $(24,25)$.

miRNAs have revealed functions in cancer biology and processes in the local microenvironment. However, only a few studies have described the effect of radiation on the miRNA expression profile and little is known about the underlying molecular mechanism (26-28). In the present study, with knowledge of the role of miRNAs in breast cancer development and different expression patterns between the breast cancer cell lines, and based on previous studies investigating the potential effect of postoperative WF on the tumor microenvironment $(8,9,29)$, it was decided to explore whether stimulation of breast cancer cells by WF can affect the expression of distinct miRNAs in cell culture.

Previous studies have revealed that miRNAs also act as potential agents for predicting radiation responses or modulating the tumor radiation response of lymphoblastic cell lines, endothelial cells and lung cancer cells $(26,27,30)$. Additionally, the dysregulation of miR-21 and miR-155 expression is associated with tumor progression (31-33). miR-21 is known as a common inflammation-inducible miRNA that targets phosphatase and tensin homolog, nuclear factor I B and pro-inflammatory programmed cell death protein $4(32,34,35)$. In breast cancer cells, miR-21 contributes to radiation resistance by compromising cell cycle progression (radiation-induced G2/M arrest) (36). miR-21 is also one of the most consistently upregulated onco-miRs in a wide range of cancers, including breast, lung or colon cancer (31). Anastasov et al (36) observed that the changes in miR-21 expression depend on the cells being radiosensitive. In the aforementioned study using two breast cancer cell lines, the authors demonstrated that miR-21 expression was significantly increased in radiation-resistant cells, but remained unchanged in the radiosensitive cell line (36). This data supports the hypothesis that regulation of miR-21 expression is not associated with oncogenesis, but rather acts as radioresistant miRNA when it is transiently overexpressed subsequent to radiation treatment (37). The present study showed that miR-21 was highly expressed in 3 of 4 analyzed breast cancer cells, consisting of the basal/mesenchymal BT-549, basal/epithelial MDA-MB-468 and luminal MCF7 cell lines. Significantly decreased expression of miR-21 was observed in the HER2-positive SK-BR3 cell line, which represents the HER2-positive histological type of breast cancer. HER 2 abnormality has a high prevalence $(22 \%)$ in breast cancer, where overexpression of HER2 is associated with an increased histological tumor grade, increased cell proliferation, cell motility, tumor invasiveness, metastases and angiogenesis, decreased apoptosis, and a poor overall prognosis $(38,39)$.

While comparing the responses of different subtypes of breast cancer to WFs obtained from patients that underwent surgery alone or surgery followed by IORT, a different profile of miR-21 was observed. The present study found that WFs have no effect on the BT-549 cell line. In MDA-MB-468 and MCF7 cells, a prominent decrease in miR-21 expression was observed. By contrast, SK-BR3 cells had increased expression of miR-21 following stimulation with WF and RT-WF. Based on the present data, it was hypothesized that both WFs, and particularly WF can increase the malignancy of SK-BR-3. Thus, the present results confirm the findings of previous studies, indicating that local recurrence after surgery is particularly common in tumors characterized by HER2 overexpression, where miR-21 is over-expressed $(40,41)$. Additionally, it was previously reported that WFs contain growth factors that induce proliferation of HER2-positive breast cancer (29).

miR-155 is also one of the most consistently upregulated onco-miRs in a wide range of cancers and it is associated with tumor progression $(31,32)$. The clinical data indicate 
that miR-155 has a crucial role in tumor development, tumor diagnosis and prognosis. It was also suggested that this miRNA promotes tumor growth, invasion and metastasis and thus, acts as an onco-miR in human cancer (42). Notably, the present results clearly indicate that the WF and RT-WF groups decreased miR-155 expression in BT-549, MDA-MB-468 and MCF7 cell lines. The SK-BR-3 cell line showed a different profile of miRNA expression in the response to WF and RT-WF. The level of miR-155 expression was 12 times higher in the WF group and 4 times higher in the RT-WF group compared with untreated cells. The present findings may contribute to the clinical response of the patients. According to Chen et al (43), decreased miR-155 expression by antisense targeting could increase the sensitivity of breast cancer cells to irradiation. Since the RT-WF group shows a decreased expression level of miR-155 compared with WF, it may be hypothesized that radiation therapy, given to the two groups of patients following surgery, may have exhibited an improved outcome in patients who had already received IORT.

These results can be highly informative, but do not provide unambiguous data to allow a simple conclusion of how surgery followed by IORT alters the tumor microenvironment. Additionally, it is important to indicate that increased miR-155 expression in breast cancer has been shown to be significantly associated with increased tumor grade, advanced tumor stage and lymph node metastasis, suggesting its potential as a clinical prognostic value (44). Shibuya et al (45) showed that increased miR-155 expression may be an effective biomarker for the prediction of poor prognosis. The present study found that in the case of SK-BR-3 stimulation by postoperative WF after IORT, the expression of miR-155 was significantly downregulated compared to the WF group. This finding may indicate that radiotherapy on the tumor bed immediately after surgery (IORT) may modulate the properties of post-operative WF and consequently change the miRNA expression. According to Jiang et al (46) overexpression of miR-155 increases the proliferation of breast cancer cell, while downregulation (using anti-miR-155) reduces the cell growth. Downregulation of miR-155, which the present study observed in RT-WF group, may then reduce the proliferation of tumor cells and finally the local recurrence of cancer. Opposite results were observed in the MCF7 cell line. The level of miR-155 expression was slightly increased in the RT-WF group compared to WF group. However, as shown by Gasparini et al (47), overexpression of miR-155 in the MCF7 cell line decreased the efficiency of homologous recombination and thus enhanced sensitivity to IR both in vivo and in vitro. This statement stays on the contrary with aforementioned study by Chen et al (48). Thus, additional data are required to better understand the key aspect on miR-155 impact on response to ionizing radiation treatment.

miR-221 has been found to be overexpressed in numerous human tumors (49-52). While analyzing breast cancer cells, Roscigno et al (53) showed that miR-221 is expressed at increased levels within the CSC population. It is also responsible for promoting tumorigenesis in triple-negative breast cancer cells by promoting EMT in those cells. Thus, patients with increased miR-221 levels show worse overall survival (54). The findings presented in the present study indicate that miR-221 expression is affected by WFs in two basal/epithelial HER2-positive and luminal subtypes of breast cancer. MDA-MB-468 and MCF7 cells show decreased expression of miR-221 subsequent to WF/RT-WF addition, with a slightly increased expression in the WF group; again, SK-BR-3 shows a different expression profile. In SK-BR-3 cells, WF statistically increases miR-221 expression (5-fold), while RT-WF only slightly stimulates its expression, without any statistical significance. As miR-221 is upregulated in the population of CSCs, we wondered whether this effect is also dependent on enriched population of CSCs. As shown in a previous study, the SK-BR-3 cell line is enriched in CSC phenotype measured by both ALDH activity and CD44/CD24-/low population after stimulation with both fluids (10). Furthermore, MCF7, MDA-MB-468 and BT-549 also represented elevated levels of CSC population after WF stimulation. However, as the present results indicate, the level of miR-221 expression was decreased in those cells. Therefore, the present study speculates, that the correlation between miR-221, CSC phenotype and WF is related to the subtype of breast cancer cell line. Additionally, it was also demonstrated that miR-221 together with miR-222 directly targets estrogen receptor alpha and overexpression of these miRNAs in breast cancer aid in the progression of the more aggressive basal-like breast cancer (55). Thus, it was hypothesized that WF may have a role in the progression of HER2-positive breast cancer, and that this effect can be partially abrogated by intraoperative radiotherapy. However, due to the preliminary nature of the current study, additional research is required to reach definitive conclusions.

Overall, the present study demonstrated that post-surgical WFs affect the expression level of selected miRNAs associated with tumor development and progression. The understanding of the crosstalk mechanism between surgery and recurrence and metastases may be particularly relevant for identifying effective treatments for breast cancer.

\section{Acknowledgements}

The present study was supported by the National Science Center, Kraków, Poland (grant no. UMO-2013/09/N/NZ4/02844).

\section{References}

1. Westlake $\mathrm{S}$ and Cooper $\mathrm{N}$ : Cancer incidence and mortality: Trends in the United Kingdom and constituent countries, 1993 to 2004. Health Stat Q, 33-46, 2008.

2. Carlson RW, Allred DC, Anderson BO, Burstein HJ, Edge SB, Farrar WB, Forero A, Giordano SH, Goldstein LJ, Gradishar WJ, et al: Metastatic breast cancer, version 1.2012: Featured updates to the NCCN guidelines. J Natl Compr Canc Netw 10: 821-829, 2012.

3. Demicheli R, Ardoino I, Boracchi P, Coradini D, Agresti R, Ferraris C, Gennaro M, Hrushesky WJ and Biganzoli E: Recurrence and mortality according to estrogen receptor status for breast cancer patients undergoing conservative surgery. Ipsilateral breast tumour recurrence dynamics provides clues for tumour biology within the residual breast. BMC Cancer 10: 656, 2010.

4. Faverly DR, Hendriks JH and Holland R: Breast carcinomas of limited extent: Frequency, radiologic-pathologic characteristics, and surgical margin requirements. Cancer 91: 647-659, 2001.

5. Bitterman A, Kessner R, Goldman I, Shiloni E and Steiner M: Intraoperative radiotherapy for breast cancer. Isr Med Assoc J 14: 256-259, 2012. 
6. Murawa P, Murawa D, Adamczyk B and Polom K: Breast cancer: Actual methods of treatment and future trends. Rep Pract Oncol Radiother 19: 165-172, 2014.

7. Sedlmayer F, Reitsamer R, Fussl C, Ziegler I, Zehentmayr F, Deutschmann H, Kopp P and Fastner G: Boost IORT in breast cancer: Body of evidence. Int J Breast Cancer 2014: 472516, 2014

8. Belletti B, Vaidya JS, D'Andrea S, Entschladen F, Roncadin M, Lovat F, Berton S, Perin T, Candiani E, Reccanello S, et al: Targeted intraoperative radiotherapy impairs the stimulation of breast cancer cell proliferation and invasion caused by surgical wounding. Clin Cancer Res 14: 1325-1332, 2008.

9. Segatto I, Berton S, Sonego M, Massarut S, Perin T, Piccoli E, Colombatti A, Vecchione A, Baldassarre G and Belletti B: Surgery-induced wound response promotes stem-like and tumor-initiating features of breast cancer cells, via STAT3 signaling. Oncotarget 5: 6267-6279, 2014.

10. Zaleska K, Suchorska WM, Przybyła A and Murawa D: Effect of surgical wound fluids after intraoperative electron radiotherapy on the cancer stem cell phenotype in a panel of human breast cancer cell lines. Oncol Lett 12: 3707-3714, 2016.

11. Kong W, He L, Coppola M, Guo J, Esposito NN, Coppola D and Cheng JQ: MicroRNA-155 regulates cell survival, growth and chemosensitivity by targeting FOXO3a in breast cancer. J Biol Chem 285: 17869-17879, 2010.

12. De Mattos-Arruda L, Bottai G, Nuciforo PG, Di Tommaso L, Giovannetti E, Peg V, Losurdo A, Pérez-Garcia J, Masci G, Corsi F, et al: MicroRNA-21 links epithelial-to-mesenchymal transition and inflammatory signals to confer resistance to neoadjuvant trastuzumab and chemotherapy in HER2-positive breast cancer patients. Oncotarget 6: 37269-37280, 2015.

13. Leung CM, Chen TW, Li SC, Ho MR, Hu LY, Liu WS, Wu TT, Hsu PC, Chang HT and Tsai KW: MicroRNA expression profiles in human breast cancer cells after multifraction and single-dose radiation treatment. Oncol Rep 31: 2147-2156, 2014.

14. Prat A and Perou CM: Deconstructing the molecular portraits of breast cancer. Mol Oncol 5: 5-23, 2011.

15. Neve RM, Chin K, Fridlyand J, Yeh J, Baehner FL, Fevr T, Clark L, Bayani N, Coppe JP, Tong F, et al: A collection of breast cancer cell lines for the study of functionally distinct cancer subtypes. Cancer Cell 10: 515-527, 2006.

16. Livak KJ and Schmittgen TD: Analysis of relative gene expression data using real-time quantitative PCR and the 2(-Delta Delta C(T)) method. Methods 25: 402-408, 2001.

17. Demicheli R, Valagussa P and Bonadonna G: Does surgery modify growth kinetics of breast cancer micrometastases? Br J Cancer 85: 490-492, 2001.

18. Fisher B, Gunduz N, Coyle J, Rudock C and Saffer E: Presence of a growth-stimulating factor in serum following primary tumor removal in mice. Cancer Res 49: 1996-2001, 1989.

19. Holmgren L, O'Reilly MS and Folkman J: Dormancy of micrometastases: Balanced proliferation and apoptosis in the presence of angiogenesis suppression. Nat Med 1: 149-153, 1995.

20. Segatto I, Berton S, Sonego M, Massarut S, Fabris L, Armenia J, Mileto M, Colombatti A, Vecchione A, Baldassarre G and Belletti B p70S6 kinase mediates breast cancer cell survival in response to surgical wound fluid stimulation. Mol Oncol 8: 766-780, 2014.

21. Segatto I, Berton S, Sonego M, Massarut S, D'Andrea S, Perin T, Fabris L, Armenia J, Rampioni G, Lovisa S, et al: Inhibition of breast cancer local relapse by targeting p70S6 kinase activity. J Mol Cell Biol 5: 428-431, 2013.

22. Fidler IJ: The organ microenvironment and cancer metastasis. Differentiation 70: 498-505, 2002.

23. Paget $S$ : The distribution of secondary growths in cancer of the breast. 1889. Lancet 133: 571-573, 1889.

24. Barcellos-Hoff MH, Park C and Wright EG: Radiation and the microenvironment-tumorigenesis and therapy. Nat Rev Cancer 5 : $867-875,2005$

25. Vaidya JS, Tobias JS, Baum M, Keshtgar M, Joseph D, Wenz F, Houghton J, Saunders C, Corica T, D'Souza D, et al: Intraoperative radiotherapy for breast cancer. Lancet Oncol 5: 165-173, 2004

26. Chaudhry MA: Real-time PCR analysis of micro-RNA expression in ionizing radiation-treated cells. Cancer Biother Radiopharm 24: 49-56, 2009.

27. Weidhaas JB, Babar I, Nallur SM, Trang P, Roush S, Boehm M, Gillespie E and Slack FJ: MicroRNAs as potential agents to alter resistance to cytotoxic anticancer therapy. Cancer Res 67: 11111-11116, 2007.

28. Maes OC, An J, Sarojini $\mathrm{H}, \mathrm{Wu} \mathrm{H}$ and Wang E: Changes in MicroRNA expression patterns in human fibroblasts after low-LET radiation. J Cell Biochem 105: 824-34, 2008.
29. Tagliabue E, Agresti R, Carcangiu ML, Ghirelli C, Morelli D, Campiglio M, Martel M, Giovanazzi R, Greco M, Balsari A and Ménard S: Role of HER2 in wound-induced breast carcinoma proliferation. Lancet 362: 527-533, 2003.

30. Kraemer A, Anastasov N, Angermeier M, Winkler K, Atkinson MJ and Moertl S: MicroRNA-mediated processes are essential for the cellular radiation response. Radiation Res 176: $575-586,2011$.

31. Yang H, Kong W, He L, Zhao JJ, O'Donnell JD, Wang J, Wenham RM, Coppola D, Kruk PA, Nicosia SV and Cheng JQ: MicroRNA expression profiling in human ovarian cancer: miR-214 induces cell survival and cisplatin resistance by targeting PTEN. Cancer Res 68: 425-433, 2008.

32. Zhu S, Wu H, Wu F, Nie D, Sheng S and Mo YY: MicroRNA-21 targets tumor suppressor genes in invasion and metastasis. Cell Res 18: 350-359, 2008.

33. Zhang JG, Wang JJ, Zhao F, Liu Q, Jiang $\mathrm{K}$ and Yang GH: MicroRNA-21 (miR-21) represses tumor suppressor PTEN and promotes growth and invasion in non-small cell lung cancer (NSCLC). Clin Chim Acta 411: 846-852, 2010.

34. Li X, Xin S, He Z, Che X, Wang J, Xiao X, Chen J and Song X: MicroRNA-21 (miR-21) post-transcriptionally downregulates tumor suppressor PDCD4 and promotes cell transformation, proliferation, and metastasis in renal cell carcinoma. Cell Physiol Biochem 33: 1631-1642, 2014

35. Fujita S, Ito T, Mizutani T, Minoguchi S, Yamamichi N, Sakurai K and Iba H: miR-21 gene expression triggered by AP-1 is sustained through a double-negative feedback mechanism. J Mol Biol 378: 492-504, 2008.

36. Anastasov N, Höfig I, Vasconcellos IG, Rappl K, Braselmann H, Ludyga N, Auer G, Aubele M and Atkinson MJ: Radiation resistance due to high expression of miR-21 and G2/M checkpoint arrest in breast cancer cells. Radiat Oncol 7: 206, 2012.

37. Iliopoulos D, Jaeger SA, Hirsch HA, Bulyk ML and Struhl K: STAT3 activation of miR-21 and miR-181b-1 via PTEN and CYLD are part of the epigenetic switch linking inflammation to cancer. Mol Cell 39: 493-506, 2010.

38. Ross JS, Slodkowska EA, Symmans WF, Pusztai L, Ravdin PM and Hortobagyi GN: The HER-2 receptor and breast cancer: Ten years of targeted anti-HER-2 therapy and personalized medicine. Oncologist 14: 320-368, 2009.

39. Slamon DJ, Godolphin W, Jones LA, Holt JA, Wong SG, Keith DE, Levin WJ, Stuart SG, Udove J, Ullrich A, et al: Studies of the HER-2/neu proto-oncogene in human breast and ovarian cancer. Science 244: 707-712, 1989.

40. Lee JA, Lee HY, Lee ES, Kim I and Bae JW: Prognostic implications of microRNA-21 overexpression in invasive ductal carcinomas of the breast. J Breast Cancer 14: 269-275, 2011.

41. Gong C, Yao Y, Wang Y, Liu B, Wu W, Chen J, Su F, Yao H and Song E: Up-regulation of miR-21 mediates resistance to trastuzumab therapy for breast cancer. J Biol Chem 286: 19127-19137, 2011.

42. Zhang CM, Zhao J and Deng HY: MiR-155 promotes proliferation of human breast cancer MCF-7 cells through targeting tumor protein 53-induced nuclear protein 1. J Biomed Sci 20: 79, 2013.

43. Chen X, Chen Y, Wang Y, Yan M, Zhang J, Liu Q, Yang H and Li J: Role of miR-155 in myasthenia gravis and effect of dexamethasone on miR-155. Zhong Nan Da Xue Xue Bao Yi Xue Ban 37: 777-782, 2012 (In Chinese).

44. Cortez MA, Welsh JW and Calin GA: Circulating microRNAs as noninvasive biomarkers in breast cancer. Recent Results Cancer Res 195: 151-161, 2012.

45. Shibuya $H$, Iinuma $H$, Shimada $R$, Horiuchi $A$ and Watanabe $T$ : Clinicopathological and prognostic value of microRNA-21 and microRNA-155 in colorectal cancer. Oncology 79: 313-320, 2010

46. Jiang S, Zhang HW, Lu MH, He XH, Li Y, Gu H, Liu MF and Wang ED: MicroRNA-155 functions as an OncomiR in breast cancer by targeting the suppressor of cytokine signaling 1 gene. Cancer Res 70: 3119-3127, 2010.

47. Gasparini P, Lovat F, Fassan M, Casadei L, Cascione L, Jacob NK, Carasi S, Palmieri D, Costinean S, Shapiro CL, et al: Protective role of miR-155 in breast cancer through RAD51 targeting impairs homologous recombination after irradiation. Proc Natl Acad Sci USA 111: 4536-4541, 2014.

48. Chen J, Wang BC and Tang JH: Clinical significance of microRNA-155 expression in human breast cancer. J Surg Oncol 106: 260-266, 2012.

49. Garofalo M, Quintavalle C, Romano G, Croce CM and Condorelli G: miR221/222 in cancer: Their role in tumor progression and response to therapy. Curr Mol Med 12: 27-33, 2012. 
50. Pineau P, Volinia S, McJunkin K, Marchio A, Battiston C, Terris B, Mazzaferro V, Lowe SW, Croce CM and Dejean A: miR-221 overexpression contributes to liver tumorigenesis. Proc Natl Acad Sci USA 107: 264-269, 2010.

51. Garofalo M, Quintavalle C, Di Leva G, Zanca C, Romano G, Taccioli C, Liu CG, Croce CM and Condorelli G: MicroRNA signatures of TRAIL resistance in human non-small cell lung cancer. Oncogene 27: 3845-3855, 2008

52. Pallante P, Visone R, Ferracin M, Ferraro A, Berlingieri MT, Troncone G, Chiappetta G, Liu CG, Santoro M, Negrini M, et al: MicroRNA deregulation in human thyroid papillary carcinomas. Endocr Relat Cancer 13: 497-508, 2006.
53. Roscigno G, Quintavalle C, Donnarumma E, Puoti I, Diaz-Lagares A, Iaboni M, Fiore D, Russo V, Todaro M, Romano G, et al: MiR-221 promotes stemness of breast cancer cells by targeting DNMT3b. Oncotarget 7: 580-592, 2016.

54. Ke J, Zhao Z, Hong SH, Bai S, He Z, Malik F, Xu J, Zhou L, Chen W, Martin-Trevino R, et al: Role of microRNA221 in regulating normal mammary epithelial hierarchy and breast cancer stem-like cells. Oncotarget 6: 3709-3721, 2015

55. Zhao JJ, Lin J, Yang H, Kong W, He L, Ma X, Coppola D and Cheng JQ: MicroRNA-221/222 negatively regulates estrogen receptor alpha and is associated with tamoxifen resistance in breast cancer. J Biol Chem 283: 31079-31086, 2008. 